Methods: The case-control pilot study included 143 children (39 with SLE and 103 healthy unrelated volunteers as a control group). Diagnosis of SLE was based on 2012 SLICC criteria. STAT4 rs7574865 G/T polymorphism was investigated using allele-specific real-time polymerase chain reaction (RT-PCR)

Results: The group of pts with SLE consisted of 29 girls and 10 boys, with an average age of $11.8 \pm 3.7$ years (from 3 to 17 years) and an average disease duration of $4.1 \pm 2.4$ years. $79.5 \%$ pts had acute cutaneous lupus at the onset, $46.1 \%$ - nonscarring alopecia, $71.8 \%$ - arthritis, $23.1 \%$ - oral and nasal ulcers, $23.1 \%$ - serositis, $43.6 \%$ - renal involvement, $35.9 \%$-neuropsychiatric disorders. Leucopenia/lymphopenia was found in $71.8 \%$ of pts, thrombocytopenia - in $23,1 \%$. ANA were detected in $100 \%$ pts, anti-dsDNA - in $79.5 \%$, anti-Sm - in $31.6 \%$, antiphospholipid antibodies - in $7,3 \%$, hypocomplementemia - in $61.5 \%$, positive direct Coombs test - in $35.9 \%$. Macrophage activation syndrome at the onset was documented in $15.4 \%$ of pts. The distribution of rs 7574865 genotypes in the control group showed no significant deviations from the Hardy-Weinberg equilibrium. The distribution of genotype frequencies among pts had statistically significant differences compared to the control $(\chi 2=12.95, p=0.0015)$ : GG-30.8\% and $63.1 \%(p=0.001)$, GT-56.4\% and $33.0 \% \quad(p=0.018)$, TT-12.8\% and $3.9 \%$ $(p=0.114)$, GT+TT $-69.2 \%$ and $36.9 \%(p=0.0005)$. The frequency of the mutant STAT 4 allele T (polymorphism), was significantly higher in the SLE group than in the control group ( $41 \%$ and $20.4 \%$, respectively; $p=0.0007)$. We identified an association of the T allele with some clinical, laboratory, and immunological disorders in SLE: arthritis (OR 3.9, $\mathrm{p}=0.0002$ ), acute cutaneous lupus (OR 2.47, $\mathrm{p}=0.003$ ), nonscarring alopecia (OR 3.12, $\mathrm{p}=0.002$ ), renal involvement (OR 2.42, $p=0.022$ ), leucopenia (OR 2.72, $p=0.003$ ), thrombocytopenia (OR 4.88, $p=0.002$ ), anti-dsDNA (OR 2.82, $p=0.0006$ ), hypocomplementemia (OR 2.34, $p=0.012$ ), positive direct Coombs test (OR 3.38, $p=0.002$ ).

Conclusion: Our pilot study confirmed that the STAT4 rs7574865 G/T polymorphism was associated with the risk of SLE in children and some of SLE manifestations.

Disclosure of Interests: None declared

DOI: 10.1136/annrheumdis-2021-eular.2142

\section{AB0008 $\quad$ APPLYING WHOLE EXOME SEQUENCING TO FAMILIAL ANTIPHOSPHOLIPID SYNDROME: NEW PLAYERS IN A RARE DISEASE?}

A. Barinotti ${ }^{1}$, M. Radin ${ }^{1}$, I. Cecchi ${ }^{1}$, S. G. Foddai ${ }^{1}$, E. Rubini ${ }^{1}$, D. Roccatello ${ }^{1}$, S. Sciascia ${ }^{1}$, E. Menegatti ${ }^{1}{ }^{1}$ University of Turin, Clinical and Biological Sciences, Turin, Italy

Background: Antiphospholipid Syndrome (APS) is an autoimmune disease whose precise aetiology is still unknown, but the high heterogeneity of its manifestations and clinical course is presumably due to the occurrence of different mechanisms and alterations at different levels and pathways [1]. The first genetic studies in APS focused primarily on the human leukocytes antigen system region, but more recent data highlighted a role of other genes in APS susceptibility, primarily those involved in the immune response and in the haemostatic process.

Objectives: We aimed to deepen the investigation of APS genetic background starting from a case of familial APS, analysing two siblings with thrombotic APS (Table 1), both triple positive for antiphospholipid antibodies (aPL).

Table 1. Main clinical and laboratory characteristics of the patients included in the study.

\begin{tabular}{|c|c|c|c|}
\hline Patient & Age & aPL Profile & Relevant Clinical History \\
\hline (F) & 51 & $\begin{array}{l}\text { Triple positive } \\
\text { (LA, aCL IgG, } \\
\text { aß2GPI IgG) }\end{array}$ & $\begin{array}{l}\text { Two episodes of ischemic stroke, one episode of CAPS } \\
\text { (renal thrombotic microangiopathy, visual impairment, } \\
\text { ischemic stroke) }\end{array}$ \\
\hline (M) & 47 & $\begin{array}{l}\text { Triple positive } \\
\text { (LA, aCL IgG, } \\
\text { aß2GPI IgG) }\end{array}$ & $\begin{array}{l}\text { Three episodes of deep vein thrombosis, regardless } \\
\text { ongoing well conducted therapy vitamin } \mathrm{k} \text { antagonist } \\
\text { and additional retinal vein thrombosis }\end{array}$ \\
\hline
\end{tabular}

LA: lupus anticoagulant; aCL: anti-cardiolipin antibodies; aß2GPI: anti- $\beta 2$ glycoprotein I antibodies; CAPS: catastrophic APS.

Methods: Genomic DNA was extracted from peripheral blood and the samples underwent Whole Exome Sequencing (WES). Sequencing was done on a $100 \mathrm{X}$ coverage, and reads have been aligned to the human reference genome (GRCh37/hg19 assembly) using the Burrows-Wheeler Alignment tool (BWA). The mean sequencing depth on target regions was 170X for patient 1, 205X for patient 2 , moreover, $99.50 \%$ of the targeted bases had at least $10 \mathrm{X}$ coverage for all the three donors. The resulting single nucleotide polymorphisms (SNPs) have been analysed through a step-by-step process based on their frequency population (using Genome Aggregation Database), their predicted effects on the protein (using VarSome) and a literature research about the genes carrying them. Moreover, genes previously associated with a pro-thrombotic tendency and with APS have been analysed in the two patients.
Results: Starting from more than 120000 SNPs for each patients, the analysis led to reduce the list of SNPs of interest to 27 missense mutations. The complete literature research regarding the genes carrying these mutations allowed to further reduce the number of selected genes, focusing on those that exert a role potentially involved in APS pathogenesis and development. In particular these genes (PLA2G6, HSPG2, BCL3, ZFAT, ATP2B2, CRTC3 and ADCY3) take part in the immune response and the vascular homeostasis. The list of the DNA missense variants of interest found in our cases of familial APS is resumed in Figure 2.

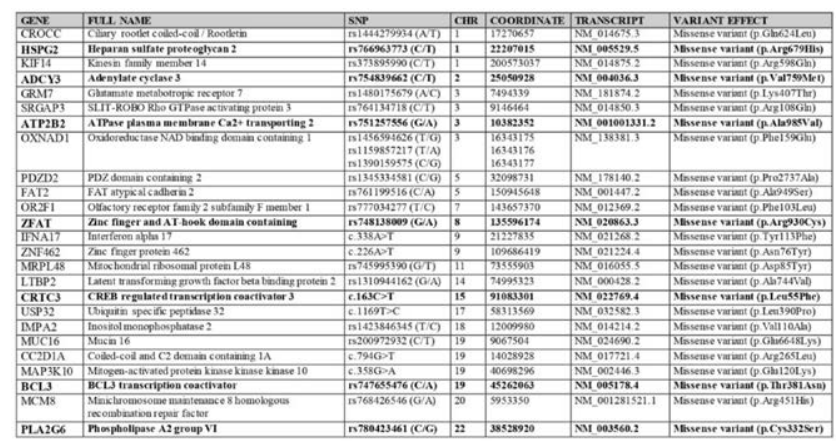

Figure 2. List of DNA missense variants of interest found in patient 1 and 2.Genes potentially involved in APS pathogenesis and development are highlighted in bold.

No mutations on genes known to be associated with a pro-thrombotic state ( $F 5$ F2, MTHFR, F13A1, PROC, PROS1, FGB and SERPINE1), or on genes previously associated with APS (B2GPI, PF4V1, SELP, TLR2, TLR4, GP Ia, GP1BA F2R, F2RL1, TFPI, F3, VEGFA, FLT1, and TNF) have been found in the WES analysis.

Conclusion: To some extent, this can be seen as a proof of concept of the complexity of APS. Efforts to interpret the genetic risk factors involved in the heterogeneous clinical features of the syndrome, for instance, the integration of WES and network-based approaches might help to identify and stratify patients at risk of developing APS.

\section{REFERENCES:}

[1] Iuliano A, Galeazzi M, Sebastiani GD. Antiphospholipid syndrome's genetic and epigenetic aspects. Autoimmun Rev. 2019;18(9).

Disclosure of Interests: None declared

DOI: 10.1136/annrheumdis-2021-eular.2499

\section{AB0009 1 ASSOCIATION OF STAT4 RS7574865, RUNX1 RS9979383, IL6 RS1800795, IL6R RS2228145, IL6R RS4845618 WITH SYSTEMIC LUPUS ERYTHEMATOSUS SUSCEPTIBILITY AND SOME LUPUS MANIFESTATIONS IN BELARUSIAN POPULATION}

N. Dostanko ${ }^{1}$, V. Yagur ${ }^{1}$, R. Goncharova ${ }^{2}$, E. Siniauskaya ${ }^{2}$, T. Zybalova $^{1}$. ${ }^{1}$ Belarusian State Medical University, 2-nd Department of Internal Medicine, Minsk, Belarus; ${ }^{2}$ National Academy of Sciences of Belarus, Institute of Genetics and Cytology, Minsk, Belarus

Background: Systemic lupus erythematosus (SLE) has a significant genetic predisposition. Many genetic variants of susceptibility to SLE have been published and analyzed, but the clinical and functional significance of the various genotypes has not yet been clearly defined [1].

Objectives: To estimate the association between some of non-HLA gene polymorphisms such as STAT4 rs7574865, RUNX1 rs9979383, IL6 rs1800795, IL6R rs2228145, IL6R rs4845618 and susceptibility to SLE in Belarusian population as well as some disease manifestations.

Methods: We examined 383 healthy blood donors and 54 SLE patients (18-72 years old, median age 35) classified according to the 1997 American College of Rheumatology (ACR) revised classification criteria [2]. Deoxyribonucleic acid was extracted from peripheral blood samples by phenol-chloroform method. Genotyping was performed by real-time PCR with fluorescent probes. Differences of distribution of all the single nucleotide polymorphism (SNP) genotypes and their associations with secondary antiphospholipid syndrom (APS) and lupus arthritis were analyzed using Pearson $\chi^{2}\left(\chi^{2}\right)$ and two-way Fisher exact test $\left(F, p_{2-t}\right)$. Diagnostic odds ratio $(\mathrm{dOR})$, likelihood ratio of positive $\left(\mathrm{LR}^{+}\right)$and negative ( $\mathrm{LR}^{-}$) tests and corresponding 95\% confidence intervals $(\mathrm{Cl})$ were also calculated

Results: We revealed significant difference in STAT4 rs7574865 genotypes in SLE patients and healthy donors $\left(\chi^{2}=8,27, p=0,016\right)$ with significant increase of TT genotype frequency in SLE patients vs healthy donors $\left(\chi^{2}=6.83 p=0.009 ; p\right.$ $=0.020 ; \mathrm{dOR}=3.78\left(\mathrm{Cl}_{95 \%} 1.36-10.55\right) ; \mathrm{LR}^{+}=3.44\left(\mathrm{Cl}_{95 \%} 1.35-8.71\right) ; \mathrm{LR}^{-}=0.91$ 